\title{
BEAM TRANSPORT OF LOW TEMPERATURE ATOMIC HYDROGEN
}

\author{
W. A. Kaufman
}

Randall Laboratory of Physics, University of Michigan, Ann Arbor, MI 48109

\section{ABSTRACT}

Analytic calculations and particle tracking simulations ${ }^{(1)}$ are presented for a polarized atomic hydrogen beam produced by extraction from an ultra-cold $(\mathrm{T}=300 \mathrm{mK}$ ) helium film coated cell in a large solenoidal magnetic field $(12 \mathrm{~T})$. Initial focusing of states 1 and 2 by the solenoidal field and subsequent focusing by a sextupole are examined within the constraints imposed by the requirements of the polarized jet for the experiments NEPTUN and NEPTUN-A at UNK.

\section{INTRODUCTION}

The transport of a low temperature $(\mathrm{T}=300 \mathrm{mK})$ electron-spin polarized hydrogen beam formed in the gradient of a large solenoidal field (12 T) and subsequently focused by a sextupole is modeled analytically. The analytic model serves as a check on the more realistic particle tracking simulation presented.

The layout of the transport system (Fig. 1) is chosen to satisfy space requirements for a weak field transition unit and $3 \mathrm{~K}$ cryopumps along the beam path.

\section{THE ANALYTIC MODEL}

The modeled system is a beam of state 1 and 2 atoms effusing from an aperture placed at $10 \mathrm{~cm}$ as measured from the magnet center. Here, the solenoid field (gradient) is $6 \mathrm{~T}(1 \mathrm{~T} / \mathrm{cm})$. The entrance face of the $20 \mathrm{~cm}$ long sextupole is placed at $35 \mathrm{~cm}$. The sextupole field is adjusted to deliver the beam to the target region at $90 \mathrm{~cm}$. For simplicity, the aperture is taken to be a monochromatic point source whose atoms' velocity $(9360 \mathrm{~cm} / \mathrm{s})$ corresponds to the average velocity in a beam effusing from a $300 \mathrm{mK}$ volume of gas.

The treatment of the solenoid field is radically simplified by setting the axial gradient to a constant. The solution to the atoms' equation of motion in the solenoid field ${ }^{[1]}$ allows the radius of the trajectory to be expressed in terms of $z$ :

$$
\rho(z)=2\left(z_{2}-z_{0}\right) \frac{\dot{\rho}_{0}}{\dot{z}_{m}} \sin \left[\frac{1}{\dot{z}_{m}}\left(\sqrt{\dot{z}_{0}^{2}+\dot{z}_{m}^{2}\left(\frac{z-z_{0}}{z_{2}-z_{0}}\right)}-\dot{z}_{0}\right)\right],
$$

where the point source at $z_{0}$ is located in a field $B_{0}$, and $z_{2}$ is where the linear field falls to zero. The axial velocity that might be gained in the solenoid gradient is

$$
\dot{z}_{m}=\sqrt{\frac{2 \mu_{B} B_{0}}{m}} .
$$

If the radial force is neglected entirely, the trajectories become parabolic.

After the solenoid field, the trajectories are straight lines that traverse a drift space, $z_{2}<z<z_{3}$, until they enter the sextupole at $z_{3}$. A sharp edge approximation is assumed, so that no longitudinal components of the sextupole field are considered. The magnitude of this field depends on $\rho$ only, 


$$
B=B_{6} \frac{\rho^{2}}{\rho_{6}^{2}},
$$

so that only a radial force acts on the atoms. $B_{6}$ and $\rho_{6}$ denote the sextupole pole-tip field and radius, respectively.

The state 1 equations of motion yield the well known result

$$
\begin{aligned}
\rho(z) & =\rho_{i} \cos \kappa\left(\frac{z-z_{i}}{\dot{z}_{i}}\right)+\frac{\dot{\rho}_{i}}{\kappa} \sin \kappa\left(\frac{z-z_{i}}{\dot{z}_{i}}\right), \\
\kappa & =2 \mu_{B} B_{6} / m \rho_{6}^{2},
\end{aligned}
$$

where the initial values $\rho_{i}, \dot{\rho}_{i}, z_{i}$, and $\dot{z}_{i}$ refer to the trajectory parameters at the sextupole entrance. The motion of state 2 atoms in a sextupole field is not as simple; the dependence of the effective magnetic moment on the field cannot be ignored. State 2 equations of motion in a sextupole field are

$$
\begin{aligned}
& \ddot{\rho}=-\kappa^{4} \rho^{3} / \sqrt{(\kappa \rho)^{4}+(a / m)^{2}}, \\
& \ddot{z}=0 .
\end{aligned}
$$

Eqn. (5a) may be integrated once to yield

$$
\dot{\rho}^{2}=\dot{\rho}_{i}^{2}+\sqrt{\left(\kappa \rho_{i}\right)^{4}+(a / m)^{2}}-\sqrt{(\kappa \rho)^{4}+(a / m)^{2}} .
$$

It is simplest to solve Eqn. 6 numerically. Use of Eqns. 1,4 and the solution of Eqn. 6, the fact that the trajectories are straight lines in the drift spaces, and matching at the boundaries between the various regions, allows a full description of the trajectories emanating from a point source at $z_{0}$ and traversing the arrangement pictured in Fig. 1. A sextupole field $B_{6}(\rho=5 \mathrm{~cm})=.473 \mathrm{~T}$ focuses the paraxial state 1 trajectories to the target at $z=90 \mathrm{~cm}$.

Fig. 2 displays state 1 trajectories emanating from a point source for several different initial angles. Two characteristics of Fig. 2 are noteworthy: the solenoid field acts to compress the trajectories by a large amount, while the sextupole focuses them, with a certain amount of resulting radial aberration at the paraxial focus. Fig. 3 displays state 1 and 2 trajectories with initial angles between $0^{\circ}$ and $30^{\circ}$, in $5^{\circ}$ increments. It was assumed that from the source to the sextupole they were identical, since an appreciable difference in the trajectories only occurs where the field is small $(B \leq 506 \mathrm{G})$ and the gradients large. At angles greater than $\sim 30^{\circ}$ the trajectories nearly coincide in the sextupole, whereas for smaller initial angles state 2 is more weakly focused than state 1. Some state 2 trajectories $\left(\theta_{0} \leq 5^{\circ}\right)$ do not return to cross the magnetic axis. Provided that only the smaller angles are accepted, the sextupole acts as a nuclear spin filter, with state 1 atoms well focused in a relatively diffuse background of state 2 atoms. 


\section{PARTICLE TRACKING SIMULATION}

Populations of atomic trajectories in the solenoid-sextupole system were computed with a tracking simulation that numerically integrates the equations of motion using the Adams-Bashforth-Moulton "predictor-corrector" method." The solution computed for the initial steps, via Runge-Kutta, is used to polynomially extrapolate (predict) the solution one step advanced; the extrapolation is then corrected using derivative information at the new point. An adaptive step size algorithm was used to keep the error within specified bounds. A substantial part of the tracking routine was based on a program written by Ellilä, Niinikoski, and Penttilä..$^{[3]}$

A realistic solenoid field and its derivatives were produced numerically on a predetermined mesh, ${ }^{(\text {) }}$ and used as input to the tracking routine. Local values of the field and its derivatives were provided by spline interpolation. The sextupole field was generated analytically within the tracking program, and a sharp edge approximation used. The sextupole field was assumed to be entirely shielded from dilution by the solenoid field.

The initial data for the trajectories were generated by taking a random uniform deviate and, where feasible, applying the transformation method ${ }^{[2]}$ to produce a random deviate with the desired distribution function. Otherwise, the rejection method ${ }^{[2]}$ was employed. Specifically, the initial positions lay randomly and uniformly within a $.25 \times .75 \mathrm{~cm}^{2}$ rectangular aperture at $z=10$ cm. The initial directions and speeds were chosen according to an effusive distribution of velocities at $T=300 \mathrm{mK}$. The resulting trajectories $(5000$ per simulation) were abandoned if they struck the effective $10 \mathrm{~cm}$ sextupole bore. A sextupole field $B_{6}(\rho=5 \mathrm{~cm})=.413 \mathrm{~T}$ maximized the state 1 tranmission to the $0.5 \times 2.0 \mathrm{~cm}^{2}$ target region located at $z=90 \mathrm{~cm}$.

Figs. 4 and 5 display hits in the target plane for simulations of state 1 and state 2 transport. The transmission of state 1 (2) was .21 (.14) to the target region. In a weak guide field the resulting nuclear polarization is .60 .

The transmission is expected to improve with the inclusion of a helium film coated mirror in the transport system.

This research was supported by the U. S. Dept. of Energy. REFERENCES

1. W. A. Kaufman, to be published in Nucl. Instr. Meth. A.

2. W.H.Press, B.P.Flannery, S.A.Teukolsky, W.T.Vetterling, Numerical Recipes, (Cambridge University Press, 1989).

3. M.Ellilä, T.O.Niinikoski, and S.Penttilä, Nucl. Instr. Meth. B14 (1986) 571.

4. See for example M.T.Menzel and H.K.Stokes, User's Guide to the Poisson/Superfish Group of Codes, Los Alamos Natl. Lab. publication LA-UR-87115 (1987).

5. See V. G. Luppov, these proceedings. 


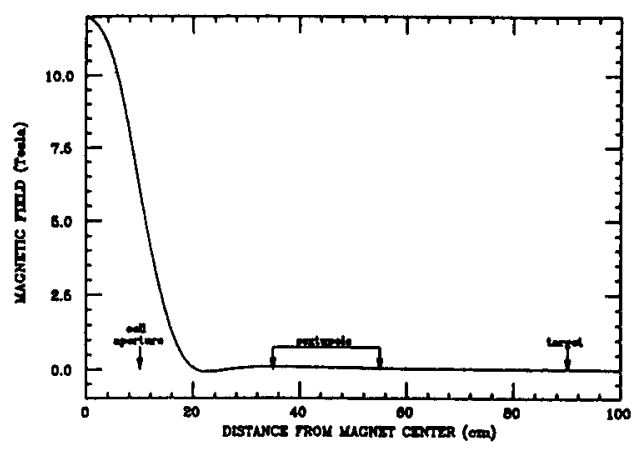

Fig. 1. The layout of the solenoid-sext upole system.

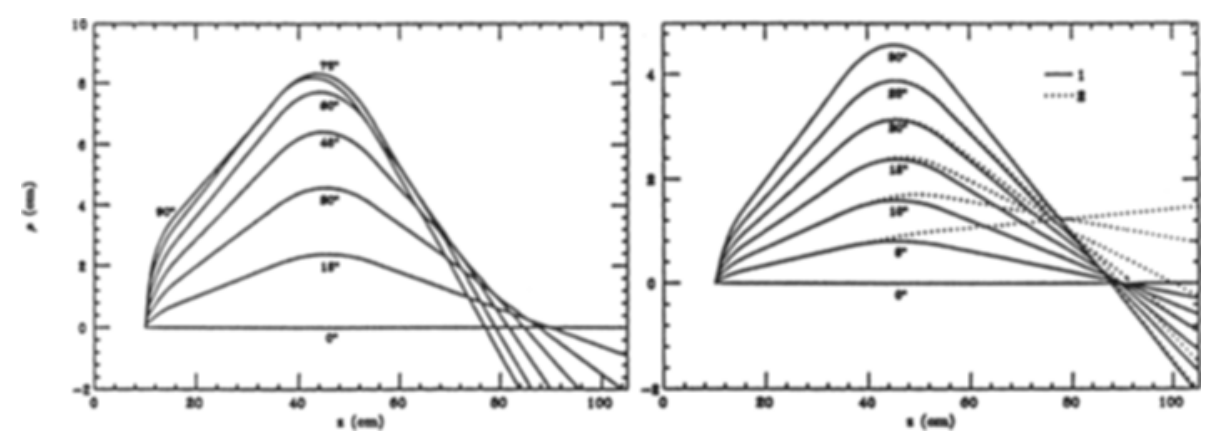

Fig. 2. State 1 trajectories' dependence on initial angle in $15^{\circ}$ increments.

Fig. 3. State 2 (dotted curves) and state 1 (solid curves) trajectories' dependence on initial angle in $5^{\circ}$ increments.



Fig. 4. Simulation of state 1 transmission to target.

Fig. 5. Simulation of state 2 transmission to target. 